\title{
Global structure of positive solutions for three-point boundary value problems
}

\author{
Jia-Ping $\mathrm{Gu}^{1}$, Liang-Gen Hu${ }^{1}$ and Huai-Nian Zhang ${ }^{2 *}$
}

\author{
"Correspondence: \\ zhanghuainian@bipt.edu.cn \\ ${ }^{2}$ Department of Mathematics and \\ Physics, Beijing Institute of \\ Petrochemical Technology, Beijing, \\ 102617, P.R. China \\ Full list of author information is \\ available at the end of the article
}

\begin{abstract}
In this paper, we are concerned with the three-point boundary value problem for second-order differential equations

$$
\left\{\begin{array}{l}
u^{\prime \prime}(t)+w(t) f(u(t))=0, \quad 0<t<1 \\
u(0)=\beta u^{\prime}(0), \quad u(1)=\alpha u(\eta)
\end{array}\right.
$$

where $\beta \geq 0,0<\eta<1,0<\alpha \eta<1$ and $1+\beta-\alpha \eta-\alpha \beta>0 ; w \in C([0,1],(0,+\infty))$ and $f \in C\left(\mathbb{R}_{+}, \mathbb{R}_{+}\right), \mathbb{R}_{+}=[0, \infty)$ satisfies $f(u)>0$ for $u>0$. The existence of the continuum of a positive solution is established by utilizing the Leray-Schauder global continuation principle. Furthermore, the interval of $\alpha$ about the nonexistence of a positive solution is also given.

MSC: 34B10; 34B18; 34G20
\end{abstract}

Keywords: positive solution; global continuous theorem; continuum; differential equation

\section{Introduction}

In this paper, we consider the following three-point boundary value problem for secondorder differential equations:

$$
\left\{\begin{array}{l}
u^{\prime \prime}(t)+w(t) f(u(t))=0, \quad 0<t<1, \\
u(0)=\beta u^{\prime}(0), \quad u(1)=\alpha u(\eta),
\end{array}\right.
$$

where $\beta \geq 0,0<\eta<1,0<\alpha \eta<1$ and $1+\beta-\alpha \eta-\alpha \beta>0 ; w \in C([0,1],(0,+\infty))$ and $f \in C\left(\mathbb{R}_{+}, \mathbb{R}_{+}\right), \mathbb{R}_{+}=[0, \infty)$ satisfies $f(u)>0$ for $u>0$.

The existence and multiplicity of positive solutions for multi-point boundary value problems have been studied by several authors and many nice results have been obtained; see, for example, [1-6] and the references therein for more information on this problem. The multi-point boundary conditions of ordinary differential equations arose in different areas of applied mathematics and physics. In addition, they are often used to model many physical phenomena which include gas diffusion through porous media, nonlinear diffusion generated by nonlinear sources, chemically reacting systems, infectious diseases as well as concentration in chemical or biological problems. In all these problems, only positive solutions are very meaningful.

C) 2013 Gu et al.; licensee Springer. This is an Open Access article distributed under the terms of the Creative Commons Attribution License (http://creativecommons.org/licenses/by/2.0), which permits unrestricted use, distribution, and reproduction in any medium, provided the original work is properly cited. 
In 2009, Sun et al. [1] studied the three-point boundary value problem

$$
\left\{\begin{array}{l}
u^{\prime \prime}(t)+\mu a(t) f(t, u(t))=0, \quad 0<t<1, \\
u(0)=\beta u^{\prime}(0), \quad u(1)=\alpha u(\eta),
\end{array}\right.
$$

where $\mu>0$ is a parameter, $\beta \geq 0,0<\eta<1,0<\alpha \eta<1$ and $1+\beta-\alpha \eta-\alpha \beta>0$. Based on Krein-Rutmann theorems and the fixed point index theory, they not only established the criteria of the existence and multiplicity of a positive solution, but also obtained the parameter $\mu$ in relation with the nonlinear term $f$ and the first eigenvalue of the linear operator.

On the other hand, we note that the nice results in [1] only gave the existence and multiplicity of positive solutions, and if the parameter $\alpha$ is regarded as a variable, then an interesting problem as to what happens to the global structure of positive solutions of (1.2) was not considered. However, this relationship is very useful for computing the numerical solution of (1.2) as it can be used to guide the numerical work. For example, the global bifurcation of solutions for second-order differential equations has been extensively studied in the literature, see $[4,7,8]$.

Motivated by this, in this paper, we consider the three-point boundary value problem for second-order differential equations (1.1) and make use of the Leray-Schauder global continuation theorem in the frame of techniques nicely employed by Ma and Thompson [4] and convex analysis technique. We consider two cases $f_{0}=0, f_{\infty}=\infty$ and $f_{0}=\infty, f_{\infty}=0$, and establish the existence of continuum of positive solutions, where $f_{0}=\lim _{u \rightarrow 0^{+}} \frac{f(u)}{u}$ and $f_{\infty}=\lim _{u \rightarrow \infty} \frac{f(u)}{u}$. Moreover, the interval of parameter $\alpha$ about the nonexistence of positive solutions is also given. Our main results extend and improve the corresponding results [1, 3 , 4]. In contrast to [1, Theorem 3.1 and Theorem 3.2], we obtain the global structure and behavior of positive solutions, where the parameter $\alpha$ is regarded as a variable.

The rest of this paper is arranged as follows. In Section 2, we give Green's function and some lemmas. In Section 3, we consider the case $f_{0}=0, f_{\infty}=\infty$, and give the existence of the continuum of positive solutions and the interval of parameter $\alpha$ about the nonexistence of positive solutions. In Section 4 , we study the case $f_{0}=\infty, f_{\infty}=0$, and give the existence of global continuum of positive solutions.

\section{Preliminaries and lemmas}

Let $X=C[0,1]$ denote the Banach space of a continuous function with the maximum norm

$$
\|u\|=\max _{t \in[0,1]}|u(t)| .
$$

Define a set by

$$
P:=\{u \in C[0,1]: u \text { is concave in }[0,1] \text { and } u(t) \geq 0\} \text {, }
$$

then $P$ is a cone.

We assume that

(H0) $\beta \geq 0,0<\eta<1,0<\alpha \eta<1$ and $\ell=1+\beta-\alpha \eta-\alpha \beta>0$. 
Lemma 2.1 (see [1, Lemma 2.1]) Suppose that condition (H0) holds and $x \in L[0,1]$. Then the following linear differential equation

$$
\begin{cases}u^{\prime \prime}(t)+x(t)=0, & 0<t<1, \\ u(0)=\beta u^{\prime}(0), & u(1)=\alpha u(\eta),\end{cases}
$$

has a unique solution

$$
u(t)=\int_{0}^{1} G(t, s) x(s) d s
$$

where $G(t, s):[0,1] \times[0,1] \rightarrow[0, \infty)$ is defined by

$$
G(t, s)=\frac{1}{\ell} \begin{cases}(s+\beta)((1-t)+\alpha(t-\eta)), & 0 \leq s \leq t \leq 1,0 \leq s \leq \eta<1 ; \\ ((s+\beta)(1-t)+\alpha(t-s)(\eta+\beta)), & 0<\eta \leq s \leq t \leq 1 ; \\ (t+\eta)((1-s)+\alpha(s-\eta)), & 0 \leq t \leq s \leq \eta<1 ; \\ (t+\beta)(1-s), & 0 \leq t \leq s \leq 1,0<\eta \leq s \leq 1 .\end{cases}
$$

For the sake of convenience, we list the following hypotheses:

(H1) $w \in C([0,1],(0,+\infty))$.

(H2) $f \in C\left(\mathbb{R}_{+}, \mathbb{R}_{+}\right)$satisfies $f(u)>0$ for $u>0$.

(H3) $f_{0}=\lim _{u \rightarrow 0^{+}} \frac{f(u)}{u}=0, f_{\infty}=\lim _{u \rightarrow \infty} \frac{f(u)}{u}=\infty$ (superlinear).

(H4) $f_{0}=\lim _{u \rightarrow 0^{+}} \frac{f(u)}{u}=\infty, f_{\infty}=\lim _{u \rightarrow \infty} \frac{f(u)}{u}=0$ (sublinear).

Lemma 2.2 Assume that $(\mathrm{H} 0)$ holds. Let $x \in C[0,1]$ with $x(t) \geq 0$ for $t \in[0,1]$ and let $u$ be a solution of

$$
\left\{\begin{array}{l}
u^{\prime \prime}(t)+x(t)=0, \quad t \in(0,1), \\
u(0)=\beta u^{\prime}(0), \quad u(1)=\alpha u(\eta) .
\end{array}\right.
$$

Then $u(t) \geq 0$ for $t \in[0,1]$. Moreover, if $x(\varsigma)>0$ for some $\varsigma \in[0,1]$, then $u(t)>0$ for all $t \in(0,1)$.

Proof We only show that if $x(\varsigma)>0$ for some $\varsigma \in[0,1]$, then $u(t)>0$ for all $t \in(0,1)$.

If $\beta=0$, then we have from [3, Lemma 2] that the results hold.

Next, we consider the case $\beta>0$. If it is not true, then there exists some $t_{1} \in[0,1]$ such that

$$
u\left(t_{1}\right)<0 .
$$

We separate the proof into two cases: Case I: $t_{1}=0$ and Case II: $t_{2} \in(0,1]$.

Case I. If $t_{1}=0$, then $u^{\prime}(0)=\frac{1}{\beta} u(0)<0$. Since $u$ is concave down in $[0,1]$, we obtain that $u^{\prime}(t)<0$ and $u(t)<0$ for all $t \in[0,1]$. Set

$$
L(t):=u(0)+u^{\prime}(0) t
$$


then we find from the boundary conditions in (2.2) that

$$
\begin{aligned}
& L(1)=u(0)+u^{\prime}(0)=(1+\beta) u^{\prime}(0), \\
& L(\eta)=u(0)+u^{\prime}(0) \eta=(\eta+\beta) u^{\prime}(0) .
\end{aligned}
$$

This together with the concavity of $u$ leads to

$$
\alpha=\frac{u(1)}{u(\eta)} \geq \frac{L(1)}{L(\eta)}=\frac{1+\beta}{\eta+\beta} .
$$

This contradicts the hypothesis $\ell=1+\beta-\alpha \eta-\alpha \beta>0$.

Case II. Consider the case $t_{1} \in(0,1]$.

(1) If $u^{\prime}(0)=0$, then $u(0)=\beta u^{\prime}(0)=0$ and the concavity of $u$ imply that

$u^{\prime}(t) \leq 0, \forall t \in[0,1]$. Hence, we get that $u(t) \leq 0, \forall t \in[0,1]$ and $u(\eta)<0$ (since $u(\eta)=0$, we have that $u(1)=0$ leads to $u \equiv 0$. This contradicts (2.3)). Again, since $u$ is concave, we have

$$
\frac{u(1)-u(0)}{1}<\frac{u(\eta)-u(0)}{\eta} \Longleftrightarrow \frac{\alpha u(\eta)}{1}<\frac{u(\eta)}{\eta}
$$

Consequently, we obtain that $\alpha>\frac{1}{\eta}$ contradicts the condition $\alpha \eta<1$.

(2) If $u^{\prime}(0)<0$, then, adopting the same proof as in Case I, we get a contradiction.

(3) If $u^{\prime}(0)>0$, then it follows that $u(0)=\beta u^{\prime}(0)>0$. In light of $u^{\prime}(0)>0$ and the concavity of $u$, we get from (2.3) that

$$
u(1)<0 \quad \text { and } \quad u(\eta)<0 \text {, }
$$

and

$$
\frac{u(1)-u\left(t_{2}\right)}{1-t_{2}}<\frac{u(\eta)-u\left(t_{2}\right)}{\eta-t_{2}}
$$

where $t_{2} \in(0, \eta)$ and $u\left(t_{2}\right)=0$. Hence, we get from the boundary condition of (2.2) that

$$
\frac{\alpha u(\eta)}{1-t_{2}} \leq \frac{u(\eta)}{\eta-t_{2}}
$$

leads to

$$
\alpha \geq \frac{1-t_{2}}{\eta-t_{2}}>\frac{1}{\eta} .
$$

This is a contradiction.

Consequently, we get from Case I and Case II that the conclusion holds.

Remark 2.1 If $u(t)$ is positive, then we know from the proof in Lemma 2.2 that $u(t)$ may only have zero point at $t=0$ and $t=1$. 
Lemma 2.3 Let $(\mathrm{H} 0)$ hold and let $u \in C^{2}\left([0,1], \mathbb{R}_{+}\right)$be a function satisfying

$$
\left\{\begin{array}{l}
u^{\prime \prime}(t) \leq 0, \quad t \in(0,1), \\
u(0)=\beta u^{\prime}(0), \quad u(1)=\alpha u(\eta)
\end{array}\right.
$$

and

$$
\|u\|=1
$$

Then there exists $\sigma \in[0,1]$ such that

$$
0 \leq u^{\prime}(\sigma) \leq \frac{1}{\eta}
$$

Proof If $\beta=0$, then from [4, Lemma 3.3] the conclusion holds.

Next, we consider the case $\beta>0$. Clearly, it is easy to see from Lemma 2.2 that $u(t)>0$ for $t \in(0,1)$.

(1) If $\alpha \in[0,1]$, then $u(\eta) \geq \alpha u(\eta)=u(1)$. This together with the concavity of $u$ yields $u^{\prime}(1) \leq 0$. Since $u^{\prime}(0)=\frac{u(0)}{\beta} \geq 0$, there exists $\sigma \in[0,1]$ such that $u^{\prime}(\sigma)=0$.

(2) If $\alpha \in\left(1, \frac{1+\beta}{\eta+\beta}\right)$, then $u(1)=\alpha u(\eta)>u(\eta)$. Consequently, there exists $t_{3} \in[\eta, 1]$ such that

$$
u\left(t_{3}\right)=\max _{t \in[0,1]} u(t)
$$

and

$$
u^{\prime}\left(t_{3}\right) \geq 0
$$

The assumption $\|u\|=1$ and the concavity of $u$ in $[0,1]$ imply that

$$
0 \leq u^{\prime}\left(t_{3}\right) \leq u^{\prime}(\eta) \leq \frac{u(\eta)}{\eta} \leq \frac{1}{\eta}
$$

This completes the proof.

From (2.1), we define an operator $T: P \rightarrow X$ as follows:

$$
T u(t):=\int_{0}^{1} G(t, s) w(s) f(u(s)) d s=: T(\alpha, u)(t) .
$$

Assume that (H0)-(H2) hold, then it is easy to verify that $T: P \rightarrow P$ is well defined and completely continuous. We note that $u$ is a positive solution of problem (1.1) if and only if $u=T(\alpha, u)$ on $P$.

By a positive solution of (1.1) we mean a solution of (1.1) which is positive on $(0,1)$.

Denote by $\mathcal{S}$ the closure of the set

$$
\left\{(\alpha, u) \in\left[0, \frac{1+\beta}{\eta+\beta}\right) \times C[0,1]: u \text { is a positive solution of }(1.1)\right\}
$$

in $\mathbb{R} \times C[0,1]$. 
Using the Leray-Schauder global continuation theorem [8, Theorem 14.C], Ma and Thompson [4, Lemma 2.2] obtained the following result.

Lemma 2.4 Let $P$ be a cone in a Banach space $X$. Let $U \subset P$ be a bounded and open subset in $X$ with respect to the topology induced by $\|\cdot\|$ on $P$. Assume that the operator $T:\left[\alpha_{1}, \alpha_{2}\right] \times P \rightarrow P$ is a continuous, compact map satisfying:

(1) the equation $u=T(\alpha, u)$ has no solution on $\left[\alpha_{1}, \alpha_{2}\right] \times(P \backslash U)$;

(2) $i\left(T\left(\alpha_{1}, \cdot\right), U, P\right)=k$ with $k \neq 0$.

Then the set

$$
\mathcal{S}^{*}=\left\{(\alpha, u) \in\left[\alpha_{1}, \alpha_{2}\right] \times P: u=T(\alpha, u)\right\}
$$

has a continuum $\mathcal{L}$ of solutions in $\left[\alpha_{1}, \alpha_{2}\right] \times P$, which connects the set $\left\{\alpha_{1}\right\} \times U$ with the set $\left\{\alpha_{2}\right\} \times U$.

\section{The superlinear case}

For a given $\alpha \in\left[0, \frac{1+\beta}{\eta+\beta}\right)$, we let

$$
\Phi_{\alpha}=\{(\alpha, u): u \in C[0,1] \text { is a nontrivial solution of }(1.1)\} .
$$

From (H0)-(H2) and Lemma 2.2, we get that

$$
\Phi_{\alpha}=\{(\alpha, u): u \in C[0,1] \text { is a positive solution of (1.1) }\} .
$$

For any $\alpha \in\left[0, \frac{1+\beta}{\eta+\beta}\right)$, we denote

$$
\Psi_{\alpha}=\{(\mu, u) \in[0, \alpha] \times C[0,1]: u \in C[0,1] \text { is a nontrivial solution of }(1.1)\} .
$$

From (H0)-(H2) and Lemma 2.2, we have that

$$
\Psi_{\alpha}=\{(\mu, u) \in[0, \alpha] \times C[0,1]: u \in C[0,1] \text { is a positive solution of }(1.1)\} .
$$

Lemma 3.1 [1, Theorem 3.2] Let conditions (H0)-(H3) hold. Then there exist two constants $r_{\alpha}$ and $R_{\alpha}$ with $r_{\alpha}<R_{\alpha}$ such that problem (1.1) has at least one positive solution $u_{\alpha}$ with $r_{\alpha}<\left\|u_{\alpha}\right\|<R_{\alpha}$. Furthermore,

$$
i\left(T(\alpha, \cdot), P_{R_{\alpha}} \backslash \bar{P}_{r_{\alpha}}, P\right)=-1,
$$

where $P_{R}=\{u \in P:\|u\|<R\}$.

Proof Since $f_{0}=\infty$ and $f_{\infty}=0$, we take $\mu=1$ in [1, Theorem 3.2] and all the conditions in [1, Theorem 3.2] are satisfied. Therefore, the conclusion holds.

Lemma 3.2 Assume that $(\mathrm{H} 0)-(\mathrm{H} 3)$ hold. Let $r_{\alpha}$ and $R_{\alpha}$ be the constants as in Lemma 3.1. Then there exists a positive number $\rho_{\alpha}$ with $\left(\rho_{\alpha}, \rho_{\alpha}^{-1}\right) \supset\left[r_{\alpha}, R_{\alpha}\right]$ such that

$$
\rho_{\alpha}<\|u\|<\rho_{\alpha}^{-1}, \quad \forall(\alpha, u) \in \Psi_{\alpha}
$$

where $\Psi_{\alpha}$ is defined by (3.2). 
Proof First we claim that if $f_{0}=0$, then there exists a positive number $\rho_{1}$ such that

$$
\left\|u_{\lambda}\right\|>\rho_{1}, \quad \forall\left(\lambda, u_{\lambda}\right) \in \Psi_{\alpha} .
$$

Suppose this fails, that is, there exists a sequence $\left\{\left(\lambda_{n}, u_{\lambda_{n}}\right)\right\} \subset \Psi_{\alpha}$ with $\left\|u_{\lambda_{n}}\right\| \rightarrow 0$ such that

$$
T\left(\lambda_{n}, u_{\lambda_{n}}\right)=u_{\lambda_{n}} .
$$

We may suppose that $\lambda_{n} \rightarrow \widetilde{\alpha}$ for some $\widetilde{\alpha} \in[0, \alpha]$. For the sake of convenience, we denote $u_{n}:=u_{\lambda_{n}}$. From (H1), (H2) and Lemma 2.2, we see that $u_{n}(t)>0$ for $t \in(0,1)$. Set $v_{n}(t):=$ $\frac{u_{n}(t)}{\left\|u_{n}\right\|}$, then we get

$$
\left\{\begin{array}{l}
v_{n}^{\prime \prime}(t)+w(t) F_{n}(t) v_{n}(t)=0, \quad 0<t<1, \\
v_{n}(0)=\beta v_{n}^{\prime}(0), \quad v_{n}(1)=\lambda_{n} v_{n}(\eta),
\end{array}\right.
$$

where

$$
F_{n}(t)= \begin{cases}\frac{f\left(u_{n}(t)\right)}{u_{n}(t)}, & t \in \Gamma:=\left\{t \in[0,1]: u_{n}(t) \neq 0\right\}, \\ 0, & t \in[0,1] \backslash \Gamma .\end{cases}
$$

From Remark 2.1, we know that the set $[0,1] \backslash \Gamma$ has at most two points $t=0$ and $t=1$. Therefore, from the hypothesis of $f_{0}=0$, it follows that $F_{n}$ is continuous in $[0,1]$ and there exists a constant $M_{1}$, independent of $n$, such that

$$
\left\|F_{n}\right\| \leq M_{1}
$$

This together with (3.4) yields

$$
\left\|v_{n}^{\prime \prime}\right\| \leq M_{1}\|w\|<\infty
$$

In light of Lemma 2.3, there exists $\sigma_{n} \in(0,1), n=1,2, \ldots$, such that

$$
0 \leq v_{n}^{\prime}\left(\sigma_{n}\right) \leq \frac{1}{\eta}, \quad n=1,2, \ldots
$$

Applying the Newton-Leibniz formula, we find

$$
v_{n}^{\prime}(t)=v_{n}^{\prime}\left(\sigma_{n}\right)+\int_{\sigma_{n}}^{t} v_{n}^{\prime \prime}(s) d s .
$$

Consequently, combining (3.5) and (3.6), we conclude that

$$
\left\|v_{n}^{\prime}\right\| \leq M_{2}
$$

for some constant $M_{2}>0$ independent of $n$. Utilizing the Ascoli-Arzela theorem, we have that $\left\{v_{n}\right\}$ is a relatively compact set on $C[0,1]$. Assume, taking a subsequence if necessary, that $v_{n} \rightarrow \widetilde{v}$ in $C[0,1]$. Then $\|\widetilde{v}\|=1$ and $\widetilde{v} \geq 0$ in $[0,1]$. 
On the other hand, from $f_{0}=0$ and the fact $\left\|u_{n}\right\| \rightarrow 0$ as $n \rightarrow \infty$, it follows that

$$
\lim _{n \rightarrow \infty} \frac{f\left(u_{n}(t)\right)}{u_{n}(t)}=0
$$

uniformly holds for all $t \in[0,1]$. From (2.4) and the fact $T\left(\lambda_{n}, u_{\lambda_{n}}\right)=u_{\lambda_{n}}$, we get

$$
v_{n}(t)=\int_{0}^{1} G(t, s) w(s) \frac{f\left(u_{n}(s)\right)}{\left\|u_{n}\right\|} d s \leq \int_{0}^{1} G(t, s) w(s) \frac{f\left(u_{n}(s)\right)}{u_{n}(s)} d s\left\|v_{n}\right\| .
$$

This together with (3.7) and the Lebesgue dominated convergence theorem, we get

$$
\widetilde{v}(t) \rightarrow 0, \quad \text { as } n \rightarrow \infty, \forall t \in[0,1]
$$

contradicts $\|\widetilde{v}\|=1$. Therefore, the claim (3.3) holds.

Next, we prove that if $f_{\infty}=\infty$, then there is a positive number $\rho_{2}$ such that

$$
\left\|u_{\lambda}\right\|<\rho_{2}, \quad \forall\left(\lambda, u_{\lambda}\right) \in \Psi_{\alpha} .
$$

Suppose on the contrary that there exists a sequence $\left\{\left(\lambda_{n}, u_{\lambda_{n}}\right)\right\} \subset \Psi_{\alpha}$ with $\left\|u_{\lambda_{n}}\right\| \rightarrow \infty$ such that

$$
T\left(\lambda_{n}, u_{\lambda_{n}}\right)=u_{\lambda_{n}}
$$

We define $u_{n}:=u_{\lambda_{n}}$. From (H0)-(H2), Lemma 2.2 and the concavity of $u_{n}$, it follows that

$$
u_{n}(t)>0 \text { for } t \in(0,1)
$$

and

$$
u_{n}(t) \geq \frac{\left\|u_{n}\right\|}{4} \text { for } t \in\left[\frac{1}{4}, \frac{3}{4}\right]
$$

Take $\tau:=\frac{6 \pi^{2}}{w}$, where $w=\min _{t \in\left[\frac{1}{4}, \frac{3}{4}\right]} w(t)$. (H3) implies that there exists a constant $R>0$ such that

$$
f(u)>\tau u \quad \text { for all } u>R .
$$

Since $\lim _{n \rightarrow \infty}\left\|u_{n}\right\|=\infty$, we find a sufficiently large $N \in \mathbb{N}$ such that

$$
\left\|u_{N}\right\|>4 R
$$

This together with (3.9) and (3.10) yields

$$
f\left(u_{N}(t)\right)>\tau u_{N}(t) \quad \text { for } t \in\left(\frac{1}{4}, \frac{3}{4}\right) .
$$


Put $\psi(t):=-\cos 2 \pi t$ for $t \in\left[\frac{1}{4}, \frac{3}{4}\right]$. Hence, we have from (3.11) that

$$
\int_{\frac{1}{4}}^{\frac{3}{4}} w(t) f\left(u_{N}(t)\right) \psi(t) d t \geq \tau \int_{\frac{1}{4}}^{\frac{3}{4}} w(t) u_{N}(t) \psi(t) d t \geq \tau w \int_{\frac{1}{4}}^{\frac{3}{4}} u_{N}(t) \psi(t) d t
$$

On the other hand, multiplying (1.1) by $\psi$ and integrating by parts, we find

$$
\begin{aligned}
\int_{\frac{1}{4}}^{\frac{3}{4}} w(t) f\left(u_{N}(t)\right) \psi(t) d t & =-\int_{\frac{1}{4}}^{\frac{3}{4}} u_{N}^{\prime \prime}(t) \psi(t) d t \\
& =-\left.u_{N}^{\prime}(t) \psi(t)\right|_{\frac{1}{4}} ^{\frac{3}{4}}+\int_{\frac{1}{4}}^{\frac{3}{4}} u_{N}^{\prime}(t) \psi^{\prime}(t) d t \\
& =-\int_{\frac{1}{4}}^{\frac{3}{4}} u_{N}(t) \psi^{\prime \prime}(t) d t+\left.u_{N}(t) \psi^{\prime}(t)\right|_{\frac{1}{4}} ^{\frac{3}{4}} \\
& \leq 4 \pi^{2} \int_{\frac{1}{4}}^{\frac{3}{4}} u_{N}(t) \psi(t) d t
\end{aligned}
$$

leads to

$$
\tau w \int_{\frac{1}{4}}^{\frac{3}{4}} u_{N}(t) \psi(t) d t \leq 4 \pi^{2} \int_{\frac{1}{4}}^{\frac{3}{4}} u_{N}(t) \psi(t) d t
$$

Then we obtain

$$
\frac{6 \pi^{2}}{w}=\tau \leq \frac{4 \pi^{2}}{w}<\frac{6 \pi^{2}}{w}
$$

This is a contradiction. Consequently, conclusion (3.8) holds.

Combining (3.3) and (3.8), we let $\rho_{\alpha}=\min \left\{\rho_{1}, \rho_{2}^{-1}\right\}$. Thus, the result holds.

Theorem 3.1 Assume that conditions (H0)-(H3) hold. Then $\mathcal{S}$ contains a continuum which joins $\{0\} \times C[0,1]$ with $\left(\frac{1+\beta}{\eta+\beta}, 0\right)$.

Proof We divide the proof into four steps.

Step 1 . We construct a continuum.

For arbitrarily given $\alpha \in\left(0, \frac{1+\beta}{\eta+\beta}\right)$, let $\rho_{\alpha}$ be as in Lemma 3.2. Define a set by

$$
U_{\alpha}:=\left\{u \in P: \rho_{\alpha}<\|u\|<\rho_{\alpha}^{-1}\right\}
$$

It follows from Lemma 3.1 and the excision property of the fixed point index that

$$
i\left(T(\alpha, \cdot), U_{\alpha}, P\right)=-1
$$

From Lemma 3.2, we know that $T(\alpha, u)=u$ has no solutions in $[0, \alpha] \times\left(P \backslash U_{\alpha}\right)$. Therefore, from Lemma 2.4, there exists a continuum $\zeta^{\alpha} \subset \Psi_{\alpha}$ which joins $\Phi_{0}$ with $\Phi_{\alpha}$. Here $\Psi_{\alpha}$ is defined by (3.2), and $\Phi_{0}$ and $\Phi_{\alpha}$ are defined by (3.1). 
Let $\mathcal{S}$ be the closure of the set

$$
\left\{\left(\alpha, u_{\alpha}\right) \in\left[0, \frac{1+\beta}{\eta+\beta}\right) \times C[0,1]: u_{\alpha} \text { is a nontrivial solution of } u=T(\alpha, u)\right\},
$$

and let

$$
\mathcal{L}=\left\{\zeta: \zeta \text { is a continuum of } \mathcal{S} \text { with } \zeta \cap \Phi_{0} \neq \emptyset\right\}
$$

Since $\zeta^{(1+\beta) / 2(\eta+\beta)} \in \mathcal{L}$, we know that $\mathcal{L} \neq \emptyset$.

Step 2. We show that there exists $\zeta \in \mathcal{L}$ satisfying

$$
\{\alpha: \exists(\alpha, u) \in \zeta\}=\left[0, \frac{1+\beta}{\eta+\beta}\right)
$$

If it is not true, then there exists $\tilde{\alpha} \in\left(0, \frac{1+\beta}{\eta+\beta}\right)$ such that

$$
\sup _{\Upsilon \in \mathcal{L}}\{\sup \{\alpha: \exists(\alpha, u) \in \Upsilon\}\}=\widetilde{\alpha} .
$$

Taking $\epsilon=\frac{1}{2}\left(\frac{1+\beta}{\eta+\beta}-\widetilde{\alpha}\right)$, it follows that $\widetilde{\alpha}+\epsilon \in\left(0, \frac{1+\beta}{\eta+\beta}\right)$. Let $\rho_{\widetilde{\alpha}+\epsilon}$ be a given number by Lemma 3.1 and the set

$$
U_{\widetilde{\alpha}+\epsilon}:=\left\{u \in P: \rho_{\widetilde{\alpha}+\epsilon}<\|u\|<\rho_{\widetilde{\alpha}+\epsilon}^{-1}\right\} .
$$

Then we know, from Lemma 3.1 and the excision property of the fixed point index, that

$$
i\left(T(0, \cdot), U_{\widetilde{\alpha}+\epsilon}, P\right)=-1
$$

From Lemma 3.2, $T(\alpha, u)=u$ has no solution in $[0, \widetilde{\alpha}+\epsilon] \times\left(P \backslash U_{\widetilde{\alpha}+\epsilon}\right)$. Again, using Lemma 2.4, we find a continuum $\zeta^{\widetilde{\alpha}+\epsilon} \subset \Psi_{\widetilde{\alpha}+\epsilon}$ which joins $\Phi_{0}$ with $\Phi_{\widetilde{\alpha}+\epsilon}$. This contradicts (3.15). Therefore, the conclusion in (3.14) holds.

Step 3. Let $\zeta$ be a continuum satisfying (3.14). We claim that

$$
\zeta \cap\left\{\left(\frac{1+\beta}{\eta+\beta}, \infty\right)\right\}=\emptyset
$$

Suppose on the contrary that there exists a sequence $\left\{\left(\alpha_{n}, u_{n}\right)\right\} \subset \zeta$ such that $\alpha_{n} \rightarrow \frac{1+\beta}{\eta+\beta}$ and $\left\|u_{n}\right\| \rightarrow \infty$. From (H0)-(H2), Lemma 2.2 and the concavity of $u_{n}$, it follows that

$$
u_{n}(t)>0 \quad \text { for } t \in(0,1)
$$

and

$$
u_{n}(t) \geq \frac{\left\|u_{n}\right\|}{4} \quad \text { for } t \in\left[\frac{1}{4}, \frac{3}{4}\right]
$$

Adopting the same proof as in the second step in Lemma 3.2, we can find a contradiction. Hence, the result in (3.16) holds. 
Step 4. Let $\zeta$ be a continuum satisfying (3.14). Next we show that

$$
\zeta \cap\left\{\frac{1+\beta}{\eta+\beta} \times C[0,1]\right\}=\left\{\left(\frac{1+\beta}{\eta+\beta}, 0\right)\right\} .
$$

If it is not true, then we have

$$
\zeta \cap\left\{\frac{1+\beta}{\eta+\beta} \times C[0,1]\right\}=\left\{\left(\frac{1+\beta}{\eta+\beta}, \widetilde{u}\right)\right\}
$$

for some $\widetilde{u} \in C[0,1] \backslash\{0\}$. Then there exists a sequence $\left\{\left(\alpha_{n}, u_{n}\right)\right\} \subset \zeta$ such that

$$
\alpha_{n} \rightarrow \frac{1+\beta}{\eta+\beta}, \quad u_{n} \rightarrow \widetilde{u}
$$

and

$$
\left\{\begin{array}{l}
\widetilde{u}^{\prime \prime}(t)+w(t) f(\widetilde{u}(t))=0, \quad 0<t<1, \\
\widetilde{u}(0)=\beta \widetilde{u}^{\prime}(0), \quad \widetilde{u}(1)=\frac{1+\beta}{\eta+\beta} \widetilde{u}(\eta) .
\end{array}\right.
$$

From conditions (H0)-(H2) and Lemma 2.2, we get that $\widetilde{u}(t)>0$ on $(0,1)$ and the graph of $\widetilde{u}$ is strictly concave down on $(0,1)$.

(1) If $\beta=0$, then we obtain from the boundary condition of (3.18) that $\widetilde{u}(0)=0$ and $\widetilde{u}(1)=\frac{1}{\eta} \widetilde{u}(\eta)$. From the strict concavity of $\widetilde{u}$, it follows that

$$
\frac{\widetilde{u}(1)}{1}<\frac{\widetilde{u}(\eta)}{\eta} \Longleftrightarrow \frac{\frac{1}{\eta} \widetilde{u}(\eta)}{1}<\frac{\widetilde{u}(\eta)}{\eta}
$$

implies

$$
\frac{1}{\eta}<\frac{1}{\eta}
$$

This is a contradiction.

(2) If $\beta>0$, then $\widetilde{u}(0)>0$ (since $\widetilde{u}(0)=0$, we know that $\widetilde{u}^{\prime}(0)=0$ and the strict concavity of $\tilde{u}$ imply $u(t)<0$ on $(0,1)$, which is a contradiction). Put

$$
L(t):=\widetilde{u}(0)+\widetilde{u}^{\prime}(0) t .
$$

Then

$$
L(1)=(1+\beta) \widetilde{u}^{\prime}(0), \quad L(\eta)=(\eta+\beta) \widetilde{u}^{\prime}(0) .
$$

From the strict concavity of $\widetilde{u}$, we get that

$$
\frac{1+\beta}{\eta+\beta}=\frac{\widetilde{u}(1)}{\widetilde{u}(\eta)}<\frac{L(1)}{L(\eta)}=\frac{1+\beta}{\eta+\beta},
$$

a contradiction.

Consequently, the conclusion in (3.17) holds. 
Remark 3.1 In contrast to [1, Theorem 3.2], we obtain the global structure and behavior of positive solutions, where the parameter $\alpha$ is regarded as a variable.

Theorem 3.2 Suppose that $\beta \geq 0, \eta \in(0,1)$ and $\alpha>\frac{1+\beta}{\eta+\beta}$. Let condition (H1) and $f \in$ $C\left(\mathbb{R}, \mathbb{R}_{+}\right)$hold, and let $u \in C^{2}[0,1]$ be a solution of

$$
\left\{\begin{array}{l}
u^{\prime \prime}(t)+w(t) f(u(t))=0, \quad t \in(0,1), \\
u(0)=\beta u^{\prime}(0), \quad u(1)=\alpha u(\eta) .
\end{array}\right.
$$

Then problem (3.19) has no positive solutions.

Proof If $f(0)=0$, then we know that $u(t) \equiv 0$ for $t \in[0,1]$ is a trivial solution of (3.19).

Suppose on the contrary that there exists a positive solution $u(t)$ to equation (3.19), i.e.,

$$
u(t)>0 \quad \text { for some } t \in(0,1) .
$$

Therefore, we know from equation (3.19) that $u^{\prime \prime}(t) \leq 0$ and $u(t)$ is concave down in $[0,1]$. Now, we consider two cases. Case I: $\beta=0$; Case II: $\beta>0$.

(1) Case I. $\beta=0$.

Clearly, we know from the boundary conditions of (3.19) that $u(0)=0$ and $\alpha>\frac{1}{\eta}$. Since $u$ is concave down and $u$ is a positive solution of equation (3.19), we obtain that

$$
\frac{u(1)-u(0)}{1} \leq \frac{u(\eta)-u(0)}{\eta}
$$

implies

$$
\frac{\alpha u(\eta)}{1} \leq \frac{u(\eta)}{\eta}
$$

i.e.,

$$
\alpha \leq \frac{1}{\eta}
$$

contradicts the condition $\alpha>\frac{1}{\eta}$.

(2) Case II. $\beta>0$.

If $u^{\prime}(0) \leq 0$, then $u(0) \leq 0$ and $u^{\prime}(t) \leq 0$ imply that $u(t) \leq 0, \forall t \in[0,1]$. This contradicts (3.20).

If

$$
u^{\prime}(0)>0 \text { and } u(0)>0 \text {, }
$$

then the concavity of $u$ implies that

$$
\frac{u(1)-u(0)}{1} \leq \frac{u(\eta)-u(0)}{\eta} \Longleftrightarrow \frac{\alpha u(\eta)-u(0)}{1} \leq \frac{u(\eta)-u(0)}{\eta}
$$


Thus

$$
(1-\alpha \eta) u(\eta) \geq(1-\eta) u(0)
$$

For $\alpha \geq \frac{1}{\eta}$, we obtain that

$$
0 \geq(1-\alpha \eta) u(\eta) \geq(1-\eta) u(0) \quad \Longrightarrow \quad u(0) \leq 0
$$

contradicts (3.21)

For $\frac{1+\beta}{\eta+\beta}<\alpha<\frac{1}{\eta}$, we have $0<(1-\alpha \eta)<1$. Since $u \in C^{2}[0,1]$ and $u^{\prime \prime}(t) \leq 0$, we get from Taylor's expansion that

$$
u(\eta)=u(0)+u^{\prime}(0) \eta+\frac{u^{\prime \prime}(\theta)}{2} \eta^{2} \leq u(0)+u^{\prime}(0) \eta
$$

where $\theta \in(0, \eta)$. Substituting (3.23) into (3.22), we find that

$$
(1-\alpha \eta)\left(u(0)+u^{\prime}(0) \eta\right) \geq(1-\alpha \eta) u(\eta) \geq(1-\eta) u(0) .
$$

From the boundary condition of (3.19), it follows that

$$
(1-\alpha \eta)(\eta+\beta) u^{\prime}(0) \geq(1-\eta) \beta u^{\prime}(0)
$$

leads to

$$
\alpha \leq \frac{1+\beta}{\eta+\beta} .
$$

This is a contradiction.

Therefore, we conclude that if $\alpha>\frac{1+\beta}{\eta+\beta}$, then problem (3.19) has no positive solutions.

\section{The sublinear case}

Lemma 4.1 [1, Theorem 3.1] Let conditions ( $\mathrm{H} 0)-(\mathrm{H} 2)$ and $(\mathrm{H} 4)$ hold. Then there exist two constants $r_{\alpha}$ and $R_{\alpha}$ with $r_{\alpha}<R_{\alpha}$ such that problem (1.1) has at least one positive solution $u_{\alpha}$ with $r_{\alpha}<\left\|u_{\alpha}\right\|<R_{\alpha}$. Furthermore,

$$
i\left(T(\alpha, \cdot), P_{R_{\alpha}} \backslash \bar{P}_{r_{\alpha}}, P\right)=1,
$$

where $P_{R}=\{u \in P:\|u\|<R\}$.

Lemma 4.2 Assume that (H0)-(H2) and (H4) hold. Let $r_{\alpha}$ and $R_{\alpha}$ be the constants as in Lemma 4.1. Then there exists a positive number $\varrho_{\alpha}$ with $\left(\varrho_{\alpha}, \varrho_{\alpha}^{-1}\right) \supset\left[r_{\alpha}, R_{\alpha}\right]$ such that

$$
\varrho_{\alpha}<\|u\|<\varrho_{\alpha}^{-1}, \quad \forall(\alpha, u) \in \Psi_{\alpha},
$$

where $\Psi_{\alpha}$ is defined by (3.2). 
Proof First, we claim that if $f_{0}=\infty$, then there exists a positive number $\varrho_{1}$ such that

$$
\left\|u_{\lambda}\right\|>\varrho_{1}, \quad \forall\left(\lambda, u_{\lambda}\right) \in \Psi_{\alpha} .
$$

Suppose this fails, that is, there exists a sequence $\left\{\left(\lambda_{n}, u_{n}\right)\right\} \subset \Psi_{\alpha}$ with $\left\|u_{n}\right\| \rightarrow 0$ such that

$$
T\left(\lambda_{n}, u_{n}\right)=u_{n}
$$

From conditions (H0)-(H2) and Lemma 2.2, it follows that

$$
u_{n}(t)>0 \text { for } t \in(0,1) .
$$

The concavity of $u_{n}$ implies

$$
\left\|u_{n}\right\| \geq u_{n}(t) \geq \frac{\left\|u_{n}\right\|}{4}>0 \quad \text { for } t \in\left[\frac{1}{4}, \frac{3}{4}\right] .
$$

Choose $\varpi:=\frac{6 \pi^{2}}{w}$, where $w=\min _{t \in\left[\frac{1}{4}, \frac{3}{4}\right]} w(t)$. In light of $(\mathrm{H} 4)$, we get that there exists a constant $r>0$ such that

$$
f(u)>\varpi u \text { for all } u<r .
$$

Since $\lim _{n \rightarrow \infty}\left\|u_{n}\right\|=0$, we get that

$$
\left\|u_{N}\right\|<r \quad \text { for sufficiently large } N
$$

implies

$$
f\left(u_{N}(t)\right)>\varpi u_{N}(t) \quad \text { for } t \in\left(\frac{1}{4}, \frac{3}{4}\right) .
$$

Adopting the same proof as in Lemma 3.2, we get a contradiction. Hence, conclusion (4.1) holds.

Now, we show that if $f_{\infty}=0$, then there exists a positive number $\varrho_{2}$ such that

$$
\left\|u_{\lambda}\right\|<\varrho_{2}, \quad \forall\left(\lambda, u_{\lambda}\right) \in \Psi_{\alpha} .
$$

Define the nondecreasing function $\tilde{f} \in C\left(\mathbb{R}_{+}, \mathbb{R}_{+}\right)$by

$$
\widetilde{f}(u)=\max _{0 \leq s \leq u} f(s) .
$$

Since $f_{\infty}=0$, it follows that

$$
\lim _{s \rightarrow \infty} \frac{\tilde{f}(s)}{s}=0 .
$$


Suppose that conclusion (4.2) fails, that is, there exists a sequence $\left\{\left(\lambda_{n}, u_{n}\right)\right\} \subset \Psi_{\alpha}$ with $\left\|u_{n}\right\| \rightarrow \infty$. We may assume that $\lambda_{n} \rightarrow \bar{\alpha} \in[0, \alpha]$. From (H0)-(H2) and Lemma 2.2, we have that

$$
u_{n}(t)>0, \quad t \in(0,1)
$$

Define $p_{n}(t):=\frac{u_{n}(t)}{\left\|u_{n}\right\|}$, which implies that

$$
\left\{\begin{array}{l}
p_{n}^{\prime \prime}(t)+w(t) \frac{f\left(u_{n}(t)\right)}{\left\|u_{n}\right\|}=0, \quad 0<t<1, \\
p_{n}(0)=\beta p_{n}^{\prime}(0), \quad p_{n}(1)=\lambda_{n} p_{n}(\eta) .
\end{array}\right.
$$

Since $\frac{f\left(u_{n}(t)\right)}{\left\|u_{n}\right\|} \leq \frac{\tilde{f}\left(u_{n}(t)\right)}{\left\|u_{n}\right\|} \leq \frac{\widetilde{f}\left(\left\|u_{n}\right\|\right)}{\left\|u_{n}\right\|}$, we get from (4.3) that

$$
\lim _{n \rightarrow \infty} \frac{f\left(u_{n}(t)\right)}{\left\|u_{n}\right\|}=0
$$

uniformly holds for $t \in[0,1]$. Again, applying the proof method as that in Lemma 3.2, we get a contradiction. Consequently, conclusion (4.2) holds.

If we let $\varrho_{\alpha}=\min \left\{\varrho_{1}, \varrho_{2}^{-1}\right\}$, then combining (4.1) and (4.2), we have that the result holds.

Theorem 4.1 Let (H0)-(H2) and (H4) hold. Then $\mathcal{S}$ contains a continuum which joins $\{0\} \times C[0,1]$ with $\left(\frac{1+\beta}{\eta+\beta}, \infty\right)$.

Proof Applying the method as in Theorem 3.1, we find from Lemma 2.2, Lemma 4.1 and Lemma 4.2 that there exists a continuum $\xi \in \mathbb{L}$ satisfying

$$
\{\alpha: \exists(\alpha, u) \in \xi\}=\left[0, \frac{1+\beta}{\eta+\beta}\right)
$$

and

$$
\xi \cap\left\{\frac{1+\beta}{\eta+\beta} \times P \backslash\{\theta\}\right\}=\emptyset .
$$

Next, we only show that

$$
\xi \cap\left\{\left(\frac{1+\beta}{\eta+\beta}, 0\right)\right\}=\emptyset .
$$

Suppose on the contrary that there exists a sequence $\left\{\left(\lambda_{n}, u_{\lambda_{n}}\right)\right\} \subset \Psi_{\alpha}$ with $\left\|u_{\lambda_{n}}\right\| \rightarrow 0$ such that

$$
T\left(\lambda_{n}, u_{\lambda_{n}}\right)=u_{\lambda_{n}} .
$$

Define $u_{n}:=u_{\lambda_{n}}$. From conditions (H0)-(H2) and Lemma 2.2, it follows that for any $t \in$ $(0,1)$,

$$
u_{n}(t)>0,
$$


and the concavity of $u_{n}$ leads to

$$
u_{n}(t) \geq \frac{\left\|u_{n}\right\|}{4}>0, \quad t \in\left[\frac{1}{4}, \frac{3}{4}\right]
$$

Take $\varpi:=\frac{6 \pi^{2}}{w}$, where $w=\min _{t \in\left[\frac{1}{4}, \frac{3}{4}\right]} w(t)$. In light of (H4), we get that there exists a constant $r>0$ such that

$$
f(u)>\varpi u \text { for all } u<r .
$$

Using the same proof as in Lemma 3.2, we get a contradiction.

Hence, the conclusion holds.

Remark 4.1 In contrast to [1, Theorem 3.1], we obtain the global structure and behavior of positive solutions, where the parameter $\alpha$ is regarded as a variable.

\section{Competing interests}

The authors declare that they have no competing interests.

\section{Authors' contributions}

All authors contributed significantly in writing this paper. All authors read and approved the final manuscript.

\section{Author details}

${ }^{1}$ Department of Mathematics, Ningbo University, Ningbo, 315211, P.R. China. ${ }^{2}$ Department of Mathematics and Physics, Beijing Institute of Petrochemical Technology, Beijing, 102617, P.R. China.

\section{Acknowledgements}

The work was supported partly by NSFC (No. 11201248), K.C. Wong Magna Fund of Ningbo University and Ningbo Natural Science Foundation (No. 2012A610031).

Received: 29 April 2013 Accepted: 8 July 2013 Published: 25 July 2013

\section{References}

1. Sun, Y, Liu, LS, Zhang, JZ, Agarwal, RP: Positive solutions of singular three-point boundary value problems for second-order differential equations. J. Comput. Appl. Math. 230, 738-750 (2009)

2. Liu, LS, Liu, BM, Wu, YH: Nontrivial solutions of $m$-point boundary value problems for singular second-order differential equations with a sign-changing nonlinear term. J. Comput. Appl. Math. 224, 373-382 (2009)

3. Ma, RY: Positive solutions of a nonlinear three-point boundary value problem. Electron. J. Differ. Equ. 34, 1-8 (1999)

4. Ma, RY, Thompson, B: Global behavior of positive solutions of nonlinear three-point boundary value problems. Nonlinear Anal. 60, 685-701 (2005)

5. Amann, H: Fixed point equations and nonlinear eigenvalue problems in ordered Banach spaces. SIAM Rev. 18, 620-709 (1976)

6. Lan, KQ, Webb, JRL: Positive solutions of semilinear differential equations with singularities. J. Differ. Equ. 148, 407-421 (1998)

7. Rynne, BR: Global bifurcation for $2 m$ th-order boundary value problems and infinitely many solutions of superlinear problems. J. Differ. Equ. 188, 461-477 (2003)

8. Zeidler, E: Nonlinear Functional Analysis, I. Fixed-Point Theorems. Springer, Berlin (1993)

doi:10.1186/1687-2770-2013-174

Cite this article as: Gu et al.: Global structure of positive solutions for three-point boundary value problems.

Boundary Value Problems 2013 2013:174. 